\title{
MAESTRI Toolkit for Industrial Symbiosis: overview, lessons learnt and implications
}

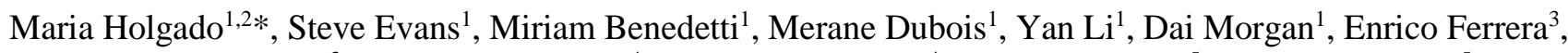 \\ Rosaria Rossini ${ }^{3}$, António J. Baptista ${ }^{4}$, Emanuel Lourenço ${ }^{4}$, Eduardo J. Silva ${ }^{5}$, Marco A. Estrela ${ }^{5}$ \\ ${ }^{1}$ Institute for Manufacturing, University of Cambridge, \\ 17 Charles Babbage Road, CB3 OFS Cambridge, UK \\ $\{$ mh769, se321, mb2132, majd3, y1483, dcm32\} dcam.ac.uk \\ ${ }^{2}$ Department of Business and Management, University of Sussex \\ Jubilee Building, BN1 9SL Brighton, UK \\ M. Holgado@sussex . ac . uk \\ ${ }^{3}$ Istituto Superiore Mario Boella, Via Pier Carlo Boggio 61, Turin, Italy \\ \{ferrera, rossini\} @ismb.it \\ ${ }^{4}$ Instituto de Engenharia Mecânica e Gestão Industrial, \\ Rua Dr. Roberto Frias, Campus da FEUP, 400 4200-465, Porto, Portugal \\ \{abaptista, elourenco\} @inegi.up.pt \\ ${ }^{5}$ Instituto de Soldadura e Qualidade, \\ Av. Prof. Dr. Cavaco Silva 33, 2740-120 Oeiras, Portugal \\ \{EJSilva, MAEstrela\}@isq.pt
}

\begin{abstract}
This paper presents a structured approach to support the development of self-organized industrial symbiosis, the Toolkit for Industrial Symbiosis. Developed within MAESTRI project, it provides a set of tools and methods to help companies gain value from wasted resources and contributes to MAESTRI goal of advancing the sustainability of European manufacturing and process industry. A participatory approach was taken for its development. The ultimate objective of this work is to encourage companies to change their attitude and consider waste as a resource and potential source for value creation.
\end{abstract}

Keywords: Industrial symbiosis, Toolkit, Eco-efficiency, Resource efficiency, Internet of Things.

\section{Introduction}

The concept of Industrial Symbiosis (IS) is one of the key areas within the Circular Economy (CE) paradigm [1] as turning waste into resources can increase resource efficiency and close material loops [2]. IS focuses on the underlying value of wasted resources within an industrial network that can be exploited through innovation and cooperation [3]. The term "wasted resources" refers here to unused or potentially reusable / recyclable energy, water and materials as well as residues and by-products of production processes. Companies within an IS industrial network establish beneficial business relationships among them based on the exchanges of these resources, e.g. Kalundborg [4]. However, these relationships are not always successful and non-technical limiting factors have been identified in relation to lack of business interest in these exchanges or creation of additional business networks, lack of trust in the new dependency links created and lack of a communication platform for the network [5]. Further difficulties (e.g. collaboration or confidentiality aspects [6]) arise when the IS exchanges emerge spontaneously from industrial interactions, i.e. without government or facilitating entities interventions, while spontaneous IS has higher potential to engage companies in long-term IS exchanges and networks.

MAESTRI is an EU-funded collaborative project (https://maestri-spire.eu/) aiming at encouraging an improvement culture by assisting the decision-making process, supporting the development of strategies and defining priorities for companies' environmental and economic performance. Its overall outcome is the MAESTRI Total Efficiency Framework (MTEF) that includes tools and methods from eco-efficiency, lean management and IS perspectives [7]. The focus of IS-oriented research within MAESTRI project is to provide companies with methods and tools to develop self-organized IS. This responds to gaps identified during a practitioneroriented review of IS concept done by Holgado et al. [3] that revealed the lack of supporting tools to identify, 
at early stages of ideation, the available opportunities for industrial symbiosis and to address the contextualization challenges of IS design and implementation. Specific support to self-organised IS included in the MTEF focuses on widening the knowledge on IS cases as a main source of information about uses of waste streams and alternative input sources, and on providing a structured, stepwise methodology for IS development, including waste identification, characterisation, valuing and exploitation [7].

This paper complements previous work [3] that reviewed the IS opportunities to assist practitioners in developing IS exchanges and networks. The aim of this paper is to provide insights on the MAESTRI approach to support self-organized IS development, including key lessons learnt during and implications for research, industry and policy.

\section{Research process}

A participatory approach [8] was taken to develop IS-oriented research activities within the MAESTRI project. An extensive literature review has been complemented with a practice review, which involved interviewing 16 companies either interested, practising or facilitating IS development, and practitioner-oriented workshops to identify key features to be integrated into the tool development process and to test concepts and development ideas (Figure 1). The understanding of IS from a practitioners' perspective [3] was central to identify focus areas and challenges to be addressed. The literature review revealed a need for companies to identify the potentially feasible IS-related opportunities for their resources and to address the contextualization challenges of IS. The support for the design and implementation of IS in different contexts, with a focus on self-organized IS, was addressed by developing a set of tools and methods for IS that are introduced in next section and described in more details in a technical report [9].

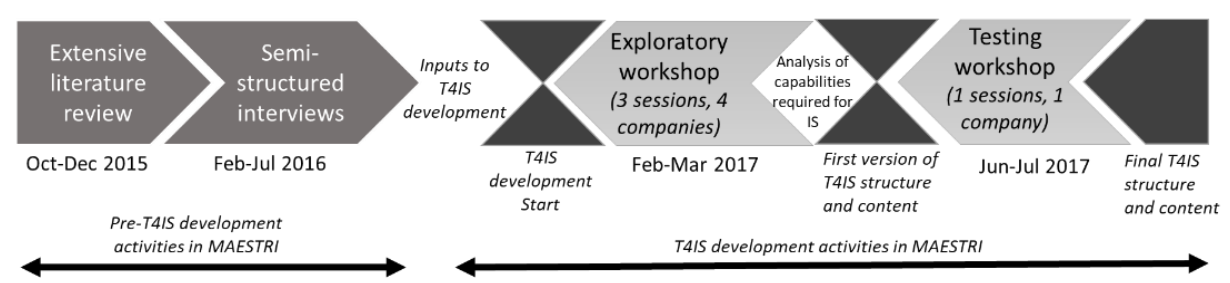

Figure 1 Overview of MAESTRI IS-oriented activities for T4IS development process

The development process was iterative with exploration and conceptualization phases continuously informing each other. One session designed during the exploratory workshop address particularly the identification of capabilities needed to develop in-company processes for IS. This session revealed six key capabilities for IS: having dedicated team for specific waste streams; leveraging on cross-functional knowledge; better time management; creating employee engagement for IS; creating networks in anticipation of new waste streams; ability to replicate good sales team for waste streams. This was taking into account to develop the first version of the toolkit. Testing and refinement phases were performed for single parts and the overall structure of the toolset (two in-company workshops provided instrumental insights) and will continue until the end of MAESTRI project. As a complement to this toolset, a user guide has been developed as a brief high-level summary for practitioners to support the understanding of IS concept and the use of the proposed set of tools and methods, thus, making them more accessible and facilitating its use by companies - enabling self-organized IS. The refinement of the user guide was discussed with MAESTRI partners, complemented with some of their ideas during its development process.

\section{Toolkit overview}

This section introduces the tools and methods that form the MAESTRI Toolkit for Industrial Symbiosis (T4IS). It represents a self-guided process that companies can use for identifying potentially exploitable wastes and value creation strategies. Although it is envisaged to need some initial training and facilitation, the process can be performed by companies alone and be certainly incorporated into their innovation processes.

The design of the T4IS allows having a standardized and structured approach to the identification and implementation of IS-based solutions for wasted resources, but at the same time to have the flexibility and adaptability 
required due to the high influence of contextual factors on the process itself. The toolkit builds on 4 guiding questions, which represents key steps to develop an IS opportunity: How to see waste; How to characterize waste; How to value waste; and How to exploit waste. The T4IS steps are briefly described herein, including primary tools and methods for each step. Expected outcomes and benefits as well as key information and participants are shown in Figure 2.

How to see waste. This step helps companies to be aware, recognize and discover wasted resources within the manufacturing process and facilities. The primary tool / method for this step is the "value uncaptured analysis for production processes". This method builds on previous work developed by Rana et al. [10] on the concept of value uncaptured. They distinguish between two types of value uncaptured: value missed and value destroyed. Previously applied to business model innovation [10] [11] and product life cycle innovation [12], we envisaged its potential application to manufacturing processes and redefined both concepts as follows: value missed refers to positive outcomes of manufacturing processes that are not adequately captured, lost or underutilized, e.g. unused capacity, dissipated heat, ineffective buffer times; and value destroyed refers to negative outcomes of manufacturing processes, e.g. waste generated, unnecessary resource consumption. Indeed, its application to manufacturing processes as part of the T4IS has confirmed its usefulness in revealing unseen wasted resources.

How to characterize waste. This step provides a method to describe the character and properties of those identified wasted resources. The primary tool / method for this step is the "MAESTRI outputs characterization method". This method consists of two sub-steps. The first one regards the categorization of secondary outputs from manufacturing processes following a systematic approach. The term "secondary outputs" refer to outputs that are not deliberately produced in manufacturing processes. The proposed categorization allows to univocally identify them according to physical properties or state and chemical properties or composition, when possible. The second one refers to gathering operational and managerial information related to those secondary outputs in an organized manner. The key additional information regards output generation rate and storage capacity, safety requirements and procedures, current disposal actions and remaining intrinsic characteristics.

How to value waste. This step helps to determine the estimated or assigned worth for the wasted resources. The primary tool / method for this step is the "IS exchange opportunities identification analysis". This method consists of three sub-steps. The first one regards performing a market analysis for those resources which market value is unknown to the company. The second one refers to searching in an IS-related repository for ideas regarding possible IS exchanges. The proposed tool here is the MAESTRI Waste Exchanges Database [13]. The third sub-step concerns scanning the company networks, supply chain or neighbouring facilities to identify initiatives regarding the wasted resources that could be replicated or could be joined as collaborator.

How to exploit waste. This step supports the generation of IS-based solutions to utilize and make the best use of the wasted materials. The primary tool / method for this step is the "IS exchange configuration analysis". This method analysis builds on two sub-steps. The first one assist the analysis of key parameters necessary for an IS exchange and the identification of any knowledge gap that needs to be fulfilled to design the exchange, e.g. whether the state, condition, volume, frequency and properties are those needed to deliver effectively the IS exchange and how to address the receiver needs for the exchangeable resource. The second one proposes the use of a well-known tool, the Business Model Canvas [14], to configure the value creation system (activities, partners, channels, etc.) to carry out the IS exchanges. 


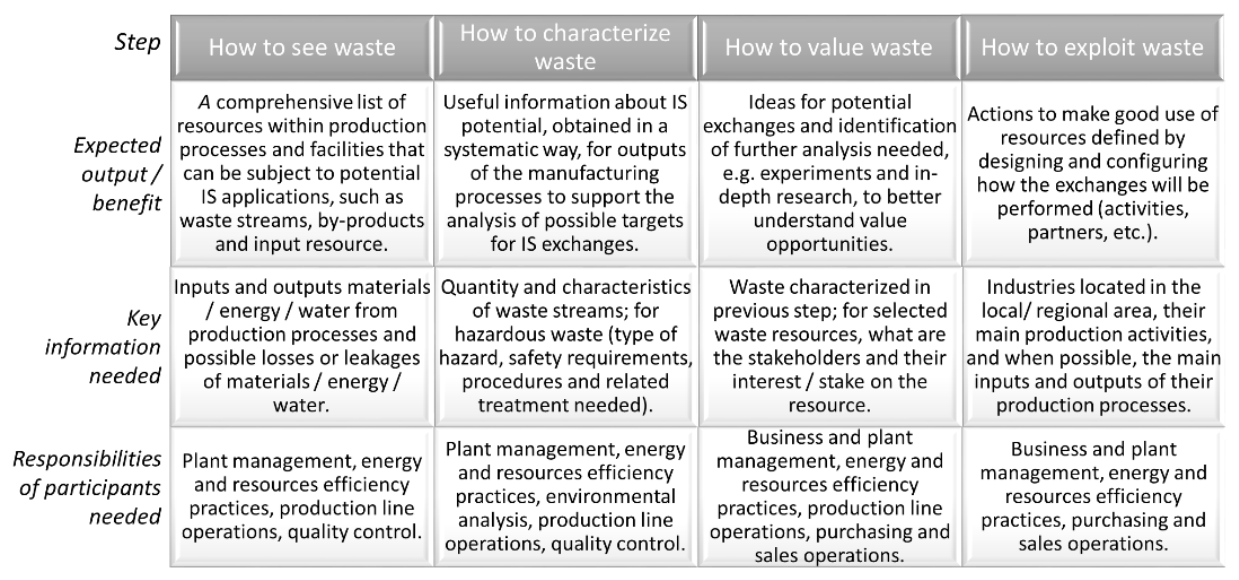

Figure 2 Expected results, input information and participants in each T4IS step

\section{Discussion}

The structure and content of the T4IS and its user guide responds to needs identified during MAESTRI ISoriented activities and specifically during the toolkit development process. They also tackle non-technical challenges identified by literature. Its developmental approach and focus on gathering multiple ideas early in the process could attract business interest on IS exchanges. The multidisciplinary character of the participants in the T4IS steps could improve the internal communication and buy-in for the IS-based solution. Bringing together cross-functional knowledge could improve the communication with external entities and potential IS partners, thus, having a positive effect on trust and confidence building among companies in the IS network.

Besides, the text and recommended actions suggested in the T4IS user guide reinforce this developmental process for companies and can lead them towards developing the necessary capabilities required to operationalise IS-based solutions. Following these recommendations and developing required capabilities using the T4IS can potentially increase the likelihood of success of IS-based solutions.

\subsection{Key lessons learnt during T4IS development}

The T4IS development process was organized into different stages with practitioner engagement activities in each of them. This allowed gathering feedback along the whole process and adapting / adjusting the design of the T4IS structure and the features of the tools and methods along the process. This approach to T4IS development provides higher confidence on the toolset results and benefits brought to companies.

The valorisation of waste is a critical step within the T4IS. This involves more than being aware of the quantities and recycling opportunities of the waste generated and brings several topics that emerged during the T4IS development activities.

As already highlighted in literature [15] [16], a lack of common language at both academic and industrial levels was detected. This could create barriers to communication and reduce the opportunities for collaboration and knowledge transfer between different sectors / industries. Efforts to create a shared MAESTRI lexicon for IS activities were increased. Besides, the use of internationally known and widely adopted codes for the categorization of waste and by-products was adopted as a means to provide the ground for a common understanding in IS exchanges information. The waste exchanges database [13] uses codes, such as EWC [17] and CPA [18], to advance towards a wider knowledge transfer of waste valorisation activities across sectors / industries to facilitate knowledge transfer to a certain extent.

The use of these codes contributes also to increase the knowledge of the waste generated and its components and to support the valorisation of waste thanks to the use of a common language. Within the T4IS, the outputs characterization method includes the use of standard codifications to help companies describe the characteristics of their wastes in a common language used and understandable by others.

Moreover, the guided brainstorming activities done during the process have proved to be fundamental to motivate people (and encourage them allocate time to these activities) and to trigger more focused feasibility studies. 


\subsection{Implications for research}

CE concepts, and more specifically IS, are driving lot of research in the Internet-of-Things (IoT) domain. Indeed, standards and technologies are highlighted as enablers of IS-based solutions implementation [19]. The development of monitoring technologies and integrated standards related to waste characteristics could enhance the quality of data available to companies for waste management and IS opportunity identification.

The creation of the waste exchanges database [13] based on standardized codes is a first step towards standardization of IS information in order to make it easily accessible, searchable and transferable among companies / sectors / industries. Further research is required to revise the proposed categorization and explore whether it could be taken as a basis for a common lexicon for IS activities.

IoT support can be envisaged in all steps of IS solutions development to some ex-tent. Waste identification and characterization steps could be enhanced by increased quality of waste data to be made available by IoT systems implemented in production processes. Besides, once the waste is identified and characterized, the IoTdomain literature proposes a huge number of infrastructures and platforms (e.g. see [20][21][22]), which can help to monitor features of interest, either real-time or historical, to communicate information whenever available only to subjects who are subscribed those specific features. This could further support the valorisation and exploitation steps, e.g. by enabling automated negotiations on a particular resource. In this regard, the literature presents several well-known algorithms that can be leveraged for automatic negotiations, such as Contract Net Proto-col [23], while many protocols are nowadays considered de facto standards for supporting secure peerto-peer communication in IoT (such as, XMPP [24], MQTT, CoAP, AMQP [25]). A use-case example can be an early and automated detection of scrap material levels achieved via interaction with the IoT plat-form. Whenever the storage area sensors detect a desired scrap level (e.g. 50\%, 75\% or 90\%), it will be annotated, reported and recorded through the IoT platform automatically. Getting accurate information about the scrap storage levels will now be possible without the need of occupying staff time to perform it manually. Real time data will help optimize the management procedures. The system will also automatically collect and compare data about the current amount per storage area in each factory. Moreover, possible IS partner companies are contacted automatically by the IoT platform, or receive continuous information on current scrap material level, enabling the establishment of an automatized electronic marketplace. Then, scrap material buyers and sellers, represented by software agents, would start an automatized bidding, allowing sellers to trade the scrap materials within the desired price range. Further research is required to investigate how companies benefit from these new functionalities to improve their IS solutions development and implementation processes. Additional research enquiries will analyse the reliance links created by these system level enablers (i.e. IoT and automatized market platforms) as well as the security of data exchange.

Additional implications of larger availability of better quality data from IoT support will also benefit manufacturing and process efficiency methodologies. The advent of a new generation of methods for efficiency assessment can provide significant support to implement IS solutions. In the context of MAESTRI and the efficiency assessment domain, methods such as MSM - Multi-Layer Stream Mapping (overall efficiency assessment) and ecoPROSYS (eco-efficiency assessment) can be very relevant tools to the T4IS, especially in wasted resources identification, quantification and characterization. By applying MSM [26] [27], it is possible to perform value and waste mapping, identifying all wastes occurring in each unit process with a lean principles basis (either for material or energy flows) and therefore providing a clear understanding and quantification of what type of residues and wastes are generated in the processes [28]. In this line, ecoPROSYS [29] [30] has a specific module for Life Cycle Assessment that defines each process unit in terms of impact and damage to the environment. This information can be used to assess and evaluate the influence of each residue and waste on the production system's environmental performance [29]. Furthermore, ecoPROSYS integrates environmental and economic dimensions to evaluate the eco-efficiency assessment of the production system, also providing relevant information for IS solutions. Further research is required to advance the understanding on the interactions between the T4IS, MSM and ecoPROSYS and on the most efficient ways to leverage on the availability of better quality data from IoT platform systems.

\subsection{Implications for industry and policy}

The T4IS steps have been tested with practitioners at the different stages of its development process. Further testing is envisaged including companies belonging to different sectors in manufacturing and process industries to introduce any necessary refinements to enhance its usability and flexibility. 
Increasing the knowledge in terms of composition, physical and chemical properties of wasted resources has been indicated by workshops participants as fundamental for uncovering the value inherent in waste. This can be especially relevant for process industries, where the waste produced is often a mix of several substances coming from different process phases. In their case, the characterization step of the T4IS is especially relevant. The use of standard codes provides a common language for cross-sectorial dialogues on IS opportunities. The T4IS help companies to identify adequate target partners and to create a stronger case for IS solutions based on their wasted resources.

Besides its direct support to IS solutions development, the use of the T4IS can support companies to understand capabilities, knowledge and trainings needs and to set appropriate development plans. It can also facilitate communication on IS solution development with potential partners, and to identify networks to build, extend, get involved in IS implementation. Indeed, the creation / strengthen of networks to share information among companies is vital to discover suitable value opportunities for wasted resources, according to the feedback received during the T4IS development.

The benefits related to establishing a common language, categorizing and coding wasted resources and increasing information sharing can be also exploited at policy and regulatory level. The creation of networks building on and getting involved in IS development can be also supported by the application of the T4IS, as a means to analyse, communicate and store information. As an example, this can support policy makers and regulators to promote the implementation of IS in specific regional areas.

\section{Concluding remarks}

While this is a well-known problem, no one before has proposed a comprehensive method to support selforganised IS development from ideation to exploitation phases.

Within MAESTRI project, the MTEF brings together several aspects for a management system in the process industry. Tools like MSM and ecoPROSYS complemented with an IS approach can enable the achievement of increased efficiency and valuable production while unlocking the inherent value of wasted resources. To support the development of self-organised IS, the MTEF includes [7]: a library of case studies and an exchanges database informing companies about possible uses for their waste streams and alternative input sources; a stepwise process to guide companies on the identification of their wasted resources, on valuing those resources and on building feasible IS-based synergies with other companies. This stepwise process, the T4IS presented here, provides a standardized and structured approach while maintaining a high degree of flexibility enabling its adaptation to different contexts.

The entire T4IS uses the term "wasted resource" as an initial attempt to change companies' perception of waste and support the mind-set shift towards seeing wastes as resources. Overall, the T4IS and its user guide have the overall goal of changing the way companies look at their waste, by considering that everything has potential to have or create value and integrating this into companies' search for competitive advantage.

Acknowledgements This work was supported by the European Union's Horizon 2020 research and innovation program (grant no. 680570).

\section{References}

1. Homrich, A.S., Galvão, G., Abadia, L.G., Carvalho, M.M.: The Circular Economy Umbrella: Trends and Gaps on Integrating Pathways. Journal of Cleaner Production, doi: 10.1016/j.jclepro.2017.11.064 (2017).

2. Jiménez-Rivero, A., García-Navarro, J.: Exploring factors influencing post-consumer gypsum recycling and landfilling in the European Union. Resources, Conservation and Recycling 116, 116-123 (2017).

3. Holgado, M., Morgan, D., Evans, S.: Exploring the scope of industrial symbiosis: Implications for practitioners. In Sustainable Design and Manufacturing 2016, pp. 169-178. Springer, Cham (2016).

4. Jacobsen, N.B.: Industrial symbiosis in Kalundborg, Denmark: a quantitative assessment of economic and environmental aspects. Journal of Industrial Ecology, 10(1-2), 239-255 (2006).

5. Sakr, D., Baas, L., El-Haggar, S., Huisingh, D.: Critical success and limiting factors for eco-industrial parks: global trends and Egyptian context. Journal of Cleaner Production, 19(11), 1158-1169 (2011).

6. Chertow, M.R.: "Uncovering” industrial symbiosis. Journal of Industrial Ecology, 11(1), 11-30 (2007). 
7. Ferrera, E., Rossini, R., Baptista, A.J., Evans, S., Hovest, G.G., Holgado, M., Lezak, E., Lourenço, E.J., Masluszczak, Z., Schneider, A. and Silva, E.J.: Toward Industry 4.0: Efficient and Sustainable Manufacturing Leveraging MAESTRI Total Efficiency Framework. In International Conference on Sustainable Design and Manufacturing, pp. 624-633. Springer, Cham, (2017)

8. Burns, D., Hyde, P., Killett, A., Poland, F., Gray, R.: Participatory organizational research: Examining voice in the coproduction of knowledge. British Journal of Management, 25(1), 133-144 (2014).

9. Holgado, M., Evans, S., Benedetti, M.: MAESTRI Deliverable 4.3. Toolkit for industrial symbiosis. Available at: https://maestri-spire.eu/downloads/technical-materials/ (2017)

10. Rana, P., Short, S., Evans, S., Holgado, M., Valkokari, K.: Toolkit for sustainable business modelling, In Value Networks in Manufacturing. Sustainability and Performance Excellence, Liyanage, Uusitalo (eds), pp. 123-153. SpringerVerlag (2017).

11. Bocken, N., Short, S., Rana, P., Evans, S.: A value mapping tool for sustainable business modelling. Corporate Governance. 13(5), 482-497 (2013)

12. Yang, M., Vladimirova, D., Rana, P., Evans, S.: Sustainable value analysis tool for value creation. Asian Journal of Management Science and Applications, 1(4), 312-332 (2014).

13. Evans, S., Benedetti, M., Holgado, M.: Library of Industrial Symbiosis case studies and linked exchanges [Dataset]. https://doi.org/10.17863/CAM.12608 (2017)

14. Osterwalder, A., Pigneur, Y.: Business model generation: a handbook for visionaries, game changers, and challengers. John Wiley \& Sons; (2010)

15. Cecelja, F., Raafat, T., Trokanas, N., Innes, S., Smith, M., Yang, A., Zorgios, Y., Korkofygas, A., Kokossis, A.: eSymbiosis: technology-enabled support for Industrial Symbiosis targeting Small and Medium Enterprises and innovation. Journal of Cleaner Production 8, 336-352 (2015).

16. Trokanas, N., Cecelja, F., Raafat, T.: Semantic input/output matching for waste pro-cessing in industrial symbiosis. Computers \& Chemical Engineering 66, 259-268 (2014).

17. European Commission: "Commission Decision on the European List of Waste", COM 2000/532/EC, available at https://goo.gl/3Zm43i, last accessed 2017/12/19. (2000)

18. European Commission: "Regulation (EC) No 451/2008 of the European Parlia-ment and of the Coucil of 23 April 2008 establishing a new statistical classification of products by activity (CPA) and repealing Council Regulation (EEC) No 3696/93", available at https://goo.gl/sfhziY, last accessed 2017/12/19. (2008)

19. Grant, G.B., Seager, T.P., Massard, G., Nies, L.: Information and communication technology for industrial symbiosis. Journal of Industrial Ecology. 14(5), 740-53 (2010)

20. Bonino, D., Alizo, M.T.D., Alapetite, A., Gilbert, T., Axling, M., Udsen, H., Soto, J.A.C., Spirito, M.: Almanac: Internet of things for smart cities. In Future Internet of Things and Cloud (FiCloud), 3rd International Conference on, pp. 309-316. IEEE (2015)

21. Kamienski, C., Jentsch, M., Eisenhauer, M., Kiljander, J., Ferrera, E., Rosengren, P., Thestrup, J., Souto, E., Andrade, W.S., Sadok, D.: Application development for the Internet of Things: A context-aware mixed criticality systems development platform. Computer Communications, 104, 1-16 (2017).

22. Brizzi, P., Lotito, A., Ferrera, E., Conzon, D., Tomasi, R., Spirito, M.: Enhancing traceability and industrial process automation through the VIRTUS middleware. In Proceedings of the Middleware 2011 Industry Track Workshop (p. 2). ACM (2011).

23. Smith, R. G.: The contract net protocol: High-level communication and control in a distributed problem solver. IEEE Transactions on computers, 12, 1104-1113 (1980)

24. Ferrera, E., Conzon, D., Brizzi, P., Rossini, R., Pastrone, C., Jentsch, M., Kool, P., Kamienski, C., Sadok, D.: XMPPbased infrastructure for IoT network management and rapid services and ap-plications development. Annals of Telecommunications, 72(7-8), 443-457 (2017)

25. Naik, N.: Choice of effective messaging protocols for IoT systems: MQTT, CoAP, AMQP and HTTP. 2017 IEEE International Systems Engineering Symposium (ISSE), Vienna, pp. 1-7 (2017)

26. Lourenço E. J., Baptista A. J., Pereira J. P., Dias-Ferreira C. Lourenço, E.J., Baptista, A.J., Pereira, J.P., Dias-Ferreira, C.: Multi-layer stream mapping as a combined approach for industrial processes eco-efficiency assessment. In Reengineering Manufacturing for Sustainability, pp. 427-433. Springer, Singapore. (2013)

27. Lourenço, E.J., Pereira, J.P., Barbosa, R., Baptista, A.J.: Using multi-layer stream mapping to assess the overall efficiency and waste of a production system: a case study from the plywood industry. Procedia CIRP, 48, 128-133 (2016)

28. Baptista, A.J., Lourenco, E.J., Pecas, P., Silva, E.J., Estrela, M.A., Holgado, M., Benedetti, M.. Evans, S.: MAESTRI Efficiency Framework as a support tool for Industrial Symbiosis implementation. Eds: Candida Vilarinho, Fernando Castro, Maria de Lurdes Lopes. CRC Press, 01/09/2017, Porto, Portugal, ISBN 978-1-351-20617-2 (2017)

29. Baptista, A.J., Lourenço, E.J., Silva, E.J., Estrela, M.A., Peças, P.: Integration of Eco-Efficiency and Efficiency Assessment Methodolo-gies: The Efficiency Framework. In International Conference on Sustainable Design and Manufacturing, pp. 613-623. Springer, Cham (2017).

30. Baptista, A.J., Lourenço, E.J., Pereira, J.P., Cunha, F., Silva, E.J. and Peças, P.: ecoPROSYS: an eco-efficiency framework applied to a medium density fiberboard finishing line. Procedia CIRP, 48, 170-175 (2016) 